ARTICLE

\title{
Comparison of Photon and Electron Absorbed Fractions in Voxel-Based and Simplified Phantoms for Small Animals
}

\author{
Akram MOHAMMADI ${ }^{*}$, Sakae KINASE and Kimiaki SAITO \\ Japan Atomic Energy Agency, 2-4 Shirane, Shirakata, Tokai-mura, Naka-gun, Ibaraki-ken, 319-1195, Japan
}

\begin{abstract}
Internal dosimetry on non-human biota is getting more important from the viewpoint of radiation protection of the environment. The International Commission on Radiological Protection (ICRP) proposed the Reference Animals and Plants using simplified phantoms, such as ellipsoids and spheres, and assessed absorbed fractions (AFs) for the whole bodies. In this study, photon and electron AFs in whole body of voxel-based frog and mouse phantoms were evaluated and compared with AFs in simplified phantoms. The evaluations were done by Monte Carlo methods for voxel-based and simplified phantoms. The monoenergetic photon or electron source was considered to be distributed uniformly in whole body. There were very small differences (less than $2 \%$ ) between whole-body AFs in voxel-based and simplified mouse phantoms, however, the differences were up to $24 \%$ for the voxel-based and the Reference Frog phantoms. Whole-body AFs in voxel-based and simplified phantoms demonstrated that not only mass but also shape of whole body affected AFs significantly. The results of this study may suggest the replacement of the Reference Animal phantoms by voxel-based animal phantoms to improve the accuracy of the whole-body AFs.
\end{abstract}

KEYWORDS: absorbed fractions, Monte Carlo, simplified phantom, voxel-based phantom

\section{Introduction}

The internal dosimetry for non-human biota is desired from the view point of environmental protection. Radiation effects assessments in non-human biota have been done for Reference Animals and Plants by the International Commission on radiological Protection (ICRP). ${ }^{1)}$ The ICRP has assessed absorbed fractions (AFs), the fraction of energy emitted by a radiation source which is absorbed within the target organ, in Reference Animals and Plants for understanding dose responses, radiation-related side-effects and the consequences of such effects. ${ }^{2)}$ The assessment has been performed only in the whole bodies of the Reference Animals for simplified dosimetric models which do not include internal organs except for the Reference Deer. ${ }^{3)}$

Besides the efforts of ICRP for radiation protection of animals, many various studies have been done on dosimetry of small animals, such as rats and mice, which are widely used in preclinical evaluations of radiopharmaceuticals. ${ }^{4-17)}$ The dose evaluations were done using the point-kernel ${ }^{4-6}$ ) and the Monte Carlo methods ${ }^{6-17)}$ in several mathematical phantoms such as stylized ${ }^{4-7)}$ and voxel-based phantoms. ${ }^{8-17)}$ The stylized phantoms are assumed to be simple shapes, such as ellipsoids, cylinders or spheroids as organs of the animals. The voxel-based phantoms were used to improve the accuracy of dosimetry since they have a more realistic anatomy and were developed from computed tomography (CT) and magnetic resonance imaging (MRI) data. AFs or S values (Gy/Bq.s), mean absorbed dose to a target organ per unit cumulated activity in the source organ, in organs of the phantoms have been assessed for organ dosimetry. One vox-

*Corresponding author, E-mail:mohammadi.akram@jaea.go.jp (C) 2011 Atomic Energy Society of Japan, All Rights Reserved. el-based frog phantom has been developed to evaluate organ doses for radiation protection of environment. ${ }^{18)}$ Photon AFs and $\mathrm{S}$ values have been evaluated for some organs of the frog phantom using the Monte Carlo simulations by Kinase. ${ }^{18,19)}$

Several voxel-based phantoms have been developed for a few small animals for dose evaluations, which can be used to assess whole-body AFs and find out how accurately the simplified phantoms can reproduce whole-body AFs. The objective of this study is comparison of whole-body AFs in voxel-based and simplified phantoms. For this purpose, AFs for both electron and photon in whole body of a voxel-based frog, voxel-based mouse, the Reference Frog and simplified mouse phantoms were evaluated with Monte Carlo simulations.

\section{Material and Methods}

\section{Validation}

AFs for photon and electron were evaluated using the Monte Carlo simulation code, EGS4, ${ }^{20)}$ in conjunction with an EGS4 user code, UCSAF. ${ }^{21)}$ In the code, the radiation transport of electron and photon in a voxel-based phantom can be simulated and correlations between primary and secondary particles are included.

The validity of our evaluations for AFs was checked directly by comparison of the AFs for monoenergetic photon and electron uniformly distributed in voxelized spheres with the results reported by Stabin. ${ }^{22)}$ AFs have been evaluated for unit density spheres in an infinite unit density with the Monte Carlo codes in the latter study. ${ }^{22)}$ For the validation of our code, two spheres with radius of 16.8 and $21.2 \mathrm{~mm}$ were converted to voxelized spheres with voxel size of $0.4 \mathrm{~mm}$ for 
the UCSAF code. Photon and electron AFs for soft tissue voxelized spheres in an infinite soft tissue medium were calculated by the code. The material composition and density $\left(1.04 \mathrm{~g} / \mathrm{cm}^{3}\right)$ of soft tissue was taken from Oak Ridge National Laboratory (ORNL) report. ${ }^{23)}$ The simulations were done for $10^{6}$ photons or electrons to reduce the fractional standard deviation to be less than $0.5 \%$.

\section{Simulation}

(1) Mouse Phantoms

The general geometry setting for "Digimouse" ${ }^{24)}$ phantom was used. The Digimouse phantom was generated using coregistered micro-CT and color cryosection images of a normal nude male mouse. This phantom was developed at the University of Southern California. A matrix of $95 \times 248 \times 52$ elements, with a voxel size of $0.4 \mathrm{~mm}$ was constructed for this study from original Digimouse with voxel size of $0.1 \mathrm{~mm}$. The organs segmented from these data are: whole brain, external cerebrum, cerebellum, olfactory bulbs, striatum, medulla, masseter muscles, eyes, lachrymal glands, heart, lungs, liver, stomach, spleen, pancreas, adrenal glands, kidneys, testes, bladder, skeleton and skin.

Because the Reference Mouse phantom has not been defined by the ICRP the simplified mouse phantom for this study was considered an ellipsoid with dimensions of $44.4 \times 15 \times 8 \mathrm{~mm}$, which converted to a voxelized ellipsoid with voxel size of $0.4 \mathrm{~mm}$. The dimensions of the ellipsoid were decided based on the dimensions, especially height, and volume of the Digimouse. The volumes of the Digimouse and simplified mouse phantoms were $22.33 \times 10^{3}$, $22.30 \times 10^{3} \mathrm{~mm}^{3}$.

(2) Frog Phantoms

The voxel-based frog phantom with the voxel size of $0.35 \mathrm{~mm}$ was reconstructed from a voxel-based frog phantom which has been developed at Japan Atomic Energy Agency. ${ }^{18)}$ The voxel size of the voxel-based frog phantom was changed from 0.35 to $0.34 \mathrm{~mm}$ to have the same volume as the Reference Frog phantom. The phantom includes 15 segmented organs: brain, blood vessel, duodenum, eyes, heart, ileum, kidneys, intestine, liver, lungs, nerve, skeleton, soft tissue, spleen and stomach.

The Reference Frog in ICRP report has been considered an ellipsoid of $80 \times 30 \times 25 \mathrm{~mm}$. The Reference Frog phantom for this study was an ellipsoid with dimensions of $80.24 \times 29.92 \times 25.16 \mathrm{~mm}^{3}$. The dimensions of the Reference Frog were slightly changed to convert it to a voxelized ellipsoid with the voxel size of $0.34 \mathrm{~mm}$. The effect of very small changes of the dimensions on the AFs is negligible.

The volumes of the frogs in the voxel-based and Reference phantoms were $31.52 \times 10^{3}$ and $31.62 \times 10^{3} \mathrm{~mm}^{3}$, respectively.

(3) Absorbed Fractions

All phantoms, including the voxel-based, Reference and simplified phantoms, were converted to input files for UCSAF code, which calculates deposited energy in the whole body of each phantom. The material composition and density of the whole bodies were assumed the same as human soft tissue and was taken from ORNL report. ${ }^{21)}$ The
Table 1 A comparison of electron AFs in spheres with volumes of $20 \times 10^{3}$ and $40 \times 10^{3} \mathrm{~mm}^{3}$ in this study with $\mathrm{Stabin}^{20}$ )

\begin{tabular}{lccc|ccc}
\hline \multicolumn{3}{l}{ Sphere : $20 \times 10^{3} \mathrm{~mm}^{3}$} & & \multicolumn{3}{|c}{ Sphere: $40 \times 10^{3} \mathrm{~mm}^{3}$} \\
\hline $\begin{array}{l}\text { Energy } \\
(\mathrm{keV})\end{array}$ & $\begin{array}{c}\text { This } \\
\text { study }\end{array}$ & Stabin & diff. \% & $\begin{array}{c}\text { This } \\
\text { study }\end{array}$ & Stabin & diff. \% \\
\hline 100 & 0.995 & 0.997 & -0.152 & 0.996 & 0.997 & -0.068 \\
200 & 0.987 & 0.989 & -0.182 & 0.990 & 0.991 & -0.116 \\
400 & 0.966 & 0.971 & -0.492 & 0.976 & 0.976 & -0.033 \\
700 & 0.940 & 0.938 & 0.160 & 0.952 & 0.952 & -0.021 \\
1000 & 0.907 & 0.904 & 0.381 & 0.926 & 0.921 & 0.509 \\
2000 & 0.799 & 0.799 & 0.019 & 0.839 & 0.830 & 1.061 \\
4000 & 0.601 & 0.589 & 1.997 & 0.676 & 0.664 & 1.713 \\
\hline
\end{tabular}

Table 2 A comparison of photon AFs in spheres with volumes of $20 \times 10^{3}$ and $40 \times 10^{3} \mathrm{~mm}^{3}$ in this study with Stabin ${ }^{20)}$

\begin{tabular}{lccc|ccc}
\hline \multicolumn{3}{l}{ Sphere : $20 \times 10^{3} \mathrm{~mm}^{3}$} & & \multicolumn{3}{|c}{ Sphere: $40 \times 10^{3} \mathrm{~mm}^{3}$} \\
\hline $\begin{array}{l}\text { Energy } \\
(\mathrm{keV})\end{array}$ & $\begin{array}{c}\text { This } \\
\text { study }\end{array}$ & Stabin & diff. \% & $\begin{array}{c}\text { This } \\
\text { study }\end{array}$ & Stabin & diff. \% \\
\hline 80 & 0.040 & 0.038 & 4.234 & 0.052 & 0.049 & 5.588 \\
100 & 0.038 & 0.036 & 4.207 & 0.049 & 0.046 & 5.393 \\
140 & 0.038 & 0.036 & 5.759 & 0.049 & 0.047 & 3.542 \\
364 & 0.042 & 0.041 & 2.959 & 0.054 & 0.052 & 3.369 \\
662 & 0.041 & 0.039 & 4.341 & 0.052 & 0.050 & 3.991 \\
1460 & 0.033 & 0.032 & 1.807 & 0.042 & 0.040 & 4.455 \\
2750 & 0.022 & 0.021 & 5.703 & 0.030 & 0.029 & 3.468 \\
\hline
\end{tabular}

masses of the whole bodies were calculated from the number of voxels of the whole bodies and the density of soft tissue. The voxel-based mouse (or frog) phantom has the same mass as the simplified mouse (or Reference Frog) phantom in order to find out how accurate the simplified phantom can reproduce absorbed fractions for the whole-body of voxel-based phantom. The source was considered to be distributed uniformly in the whole bodies with isotropic direction emission. Mono-energetic photon or electron particles were simulated in the whole bodies. Photon and electron energies were $10,15,20,30,50,100,200,500$, 1000,2000 and $4000 \mathrm{keV}$.

The photon and electron cut-offs energy were set to $1 \mathrm{keV}$ and the simulations were done for sufficient particle histories (nps $=10^{7}$ ) to reduce fractional standard deviation to be less than $5 \%$. The photon and electron cross section data were taken from PHOTX ${ }^{25)}$ and ICRU report $37,{ }^{26,27)}$ respectively.

\section{Results and Discussion}

\section{Validation}

The comparison between AFs evaluated by UCSAF code for two voxelized spheres and the data reported by Stabin ${ }^{20)}$ are presented in Tables $\mathbf{1}$ and $\mathbf{2}$ for electron and photon, respectively.

The small differences $(<6 \%)$ are attributed to different material compositions and different cross section data in two studies. The comparison reveals good agreement between the data and validates our evaluation method. 
Table 3 Photon AFs in whole body of simplified mouse and Digimouse phantoms

\begin{tabular}{lccc}
\hline Energy $(\mathrm{keV})$ & Simplified mouse & Digimouse & Diff. \% \\
\hline 10 & $8.39 \times 10^{-1}$ & $8.33 \times 10^{-1}$ & 0.77 \\
15 & $6.41 \times 10^{-1}$ & $6.36 \times 10^{-1}$ & 0.90 \\
20 & $4.03 \times 10^{-1}$ & $3.99 \times 10^{-1}$ & 0.85 \\
30 & $1.57 \times 10^{-1}$ & $1.55 \times 10^{-1}$ & 0.87 \\
50 & $4.82 \times 10^{-2}$ & $4.78 \times 10^{-2}$ & 0.79 \\
100 & $2.87 \times 10^{-2}$ & $2.84 \times 10^{-2}$ & 1.03 \\
200 & $3.27 \times 10^{-2}$ & $3.23 \times 10^{-2}$ & 1.03 \\
500 & $3.53 \times 10^{-2}$ & $3.50 \times 10^{-2}$ & 0.90 \\
1000 & $3.12 \times 10^{-2}$ & $3.08 \times 10^{-2}$ & 1.26 \\
2000 & $2.24 \times 10^{-2}$ & $2.21 \times 10^{-2}$ & 1.33 \\
4000 & $1.27 \times 10^{-2}$ & $1.26 \times 10^{-2}$ & 1.26 \\
\hline
\end{tabular}

Table 4 Electron AFs in whole body of simplified mouse and Digimouse phantoms

\begin{tabular}{lccc}
\hline Energy $(\mathrm{keV})$ & Simplified mouse & Digimouse & Diff. \% \\
\hline 10 & $1.00 \times 10^{0}$ & $1.00 \times 10^{0}$ & 0.00 \\
15 & $1.00 \times 10^{0}$ & $1.00 \times 10^{0}$ & 0.00 \\
20 & $1.00 \times 10^{0}$ & $1.00 \times 10^{0}$ & 0.00 \\
30 & $9.99 \times 10^{-1}$ & $9.99 \times 10^{-1}$ & 0.01 \\
50 & $9.99 \times 10^{-1}$ & $9.98 \times 10^{-1}$ & 0.01 \\
100 & $9.95 \times 10^{-1}$ & $9.95 \times 10^{-1}$ & 0.05 \\
200 & $9.85 \times 10^{-1}$ & $9.84 \times 10^{-1}$ & 0.13 \\
500 & $9.44 \times 10^{-1}$ & $9.40 \times 10^{-1}$ & 0.48 \\
1000 & $8.69 \times 10^{-1}$ & $8.60 \times 10^{-1}$ & 1.04 \\
2000 & $7.26 \times 10^{-1}$ & $7.15 \times 10^{-1}$ & 1.47 \\
4000 & $4.95 \times 10^{-1}$ & $4.90 \times 10^{-1}$ & 0.95 \\
\hline
\end{tabular}

\section{Simulation}

Absorbed fractions (AFs) for monoenergetic photons and electrons were calculated in the whole bodies of all phantoms as the source was distributed uniformly in the whole bodies.

(1) Absorbed Fractions in Mouse Phantoms

AFs in the whole body of Digimouse and simplified mouse phantoms are shown in Tables $\mathbf{3}$ and $\mathbf{4}$ for photons and electrons, respectively.

Differences between photon (or electron) AFs in two phantoms are negligible, i.e., they are less than $2 \%$ for all energies. From the tables it can be obtained that Digimouse and the simplified mouse phantoms, with almost the same mass, are equivalent in terms of whole-body AFs. This is due to the fact that Digimouse has a whole-body shape close to an ellipsoid.

Figure 1 compares electron/photon AFs in the whole body of MOBY phantom ${ }^{11)}$ with those observed in this study for Digimouse phantom. The comparison is favorable because the differences are attributed to differences in the whole body volumes (or masses) and geometries. The MOBY phantom is a voxel-based phantom with the volume of $33.55 \times 10^{3} \mathrm{~mm}^{3}$, which is the dominant reason for differences between AFs in the MOBY and Digimouse phantoms in Fig. 1.

(2) Absorbed Fractions in Frog Phantoms

Photon and electron AFs in the whole body of voxel-based frog and the Reference Frog phantoms are shown in Tables 5 and 6.

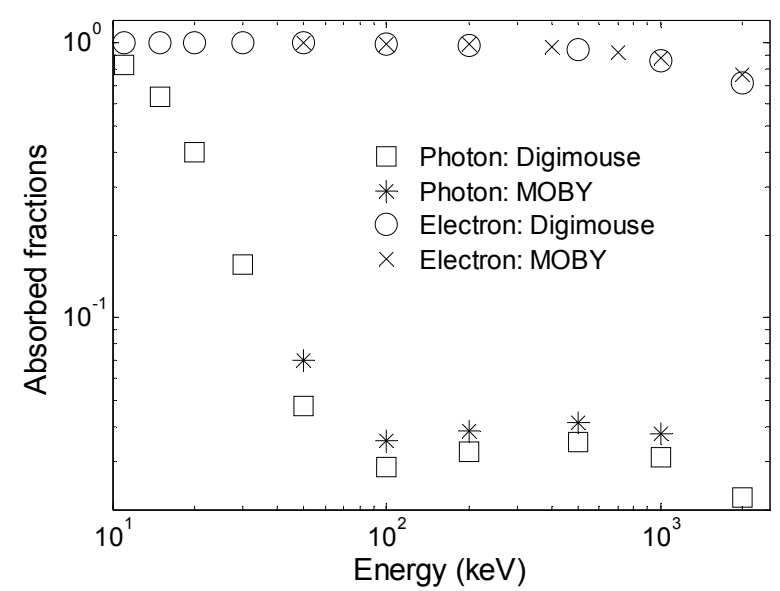

Fig. 1 A comparison of electron/photon AFs in the whole body of Digimouse and MOBY phantoms

Table 5 Photon AFs in whole body of the Reference Frog and voxel-based frog phantoms

\begin{tabular}{lccc}
\hline Energy $(\mathrm{keV})$ & Reference Frog & Voxel-based frog & Diff. \% \\
\hline 10 & $8.76 \times 10^{-1}$ & $8.24 \times 10^{-1}$ & 5.84 \\
15 & $7.07 \times 10^{-1}$ & $6.29 \times 10^{-1}$ & 11.06 \\
20 & $4.70 \times 10^{-1}$ & $3.99 \times 10^{-1}$ & 15.05 \\
30 & $1.93 \times 10^{-1}$ & $1.58 \times 10^{-1}$ & 17.80 \\
50 & $6.04 \times 10^{-2}$ & $4.92 \times 10^{-2}$ & 18.52 \\
100 & $3.56 \times 10^{-2}$ & $2.93 \times 10^{-2}$ & 17.68 \\
200 & $4.03 \times 10^{-2}$ & $3.34 \times 10^{-2}$ & 17.05 \\
500 & $4.35 \times 10^{-2}$ & $3.61 \times 10^{-2}$ & 16.99 \\
1000 & $3.89 \times 10^{-2}$ & $3.19 \times 10^{-2}$ & 17.98 \\
2000 & $2.88 \times 10^{-2}$ & $2.29 \times 10^{-2}$ & 20.55 \\
4000 & $1.74 \times 10^{-2}$ & $1.33 \times 10^{-2}$ & 23.52 \\
\hline
\end{tabular}

Table 6 Electron AFs in whole body of the Reference Frog and voxel-based frog phantoms

\begin{tabular}{lccc}
\hline Energy $(\mathrm{keV})$ & Reference Frog & Voxel-based frog & Diff. \% \\
\hline 10 & $1.00 \times 10^{0}$ & $1.00 \times 10^{0}$ & 0.00 \\
15 & $1.00 \times 10^{0}$ & $1.00 \times 10^{0}$ & 0.01 \\
20 & $1.00 \times 10^{0}$ & $1.00 \times 10^{0}$ & 0.01 \\
30 & $1.00 \times 10^{0}$ & $9.99 \times 10^{-1}$ & 0.03 \\
50 & $9.99 \times 10^{-1}$ & $9.98 \times 10^{-1}$ & 0.06 \\
100 & $9.96 \times 10^{-1}$ & $9.94 \times 10^{-1}$ & 0.19 \\
200 & $9.88 \times 10^{-1}$ & $9.82 \times 10^{-1}$ & 0.61 \\
500 & $9.57 \times 10^{-1}$ & $9.35 \times 10^{-1}$ & 2.35 \\
1000 & $9.00 \times 10^{-1}$ & $8.50 \times 10^{-1}$ & 5.45 \\
2000 & $7.87 \times 10^{-1}$ & $6.98 \times 10^{-1}$ & 11.27 \\
4000 & $5.90 \times 10^{-1}$ & $4.80 \times 10^{-1}$ & 18.65 \\
\hline
\end{tabular}

Electron AFs in the voxel-based and Reference frog phantoms are almost the same for electrons with energy less than $500 \mathrm{keV}$ because electron range is not comparable with the dimensions of the phantoms. Differences between photon AFs in two latter phantoms are $24 \%$ at maximum, while for electron they are less than $19 \%$. The whole-body shape is the most important parameter which can influence the AFs since the two phantoms have the same masses. Therefore, AFs strongly depend on the whole-body shape and considering only the mass as a parameter which can affect AFs causes large uncertainty. 


\section{Conclusion}

Absorbed fractions for both photon and electron in the whole body of voxel-based mouse and frog phantoms were compared with the AFs in the simplified mouse and the Reference Frog phantoms. The whole-body AFs in Digimouse and simplified mouse phantoms were almost identical because the whole-body shape of the Digimouse phantom was close to the geometry of simplified mouse phantom, ellipsoid. The AFs in the voxel-based and the Reference frog phantoms were different by $24 \%$, which demonstrated the strong effect of the whole-body shapes on the AFs. The whole-body shape impacted on AFs significantly, which may support the idea for the replacement of the Reference Animal phantoms by voxel-based animal phantoms to improve the accuracy of the whole-body AFs.

\section{Acknowledgment}

The authors would like to thank Dr. Ishioka, the group leader of medical radioisotope application group, Japan Atomic Energy Agency, for her support to this work.

\section{References}

1) A. Ulanovsky, G. Prohl, "Tables of dose conversion coefficients for estimating internal and external radiation exposures to terrestrial and aquatic biota," Radiat. Environ. Biophys., 47[2], 195-203 (2008).

2) J. Vives i Batlle, M. Balonov, K. Beaugelin-Seiller et al., "Inter-comparison of absorbed dose rates for non-human biota," Radiat. Environ. Biophys., 46, 349-373 (2007).

3) ICRP, "ICRP publication 108: Environmental protection: the concept and use of reference animals and plants," Ann. ICRP, 38[4-6] (2009).

4) T. E. Hui, D. R. Fisher, J. A. Kuhn, L. E. Williams, C. Nourigat, C. C. Badger, B. G. Beatty, J. D. Beatty, "A mouse model for calculating cross-organ beta-doses from yttrium-90-labeled immunoconjugates," Cancer, 73, 951-957 (1994).

5) K. S. Kolbert, T. Watson, C. Matei, S. Xu, J. A. Koutcher, G. Sgouros, "Murine S factors for liver, spleen, and kidney," $J$. Nucl. Med., 44, 784-791 (2003).

6) J. Lazewatsky, Y. Ding, D. Onthank, P. Silva, E. Solon, S. Robinson, "Radiation Dose to Abdominal Organs of the Mouse due to ${ }^{90} \mathrm{Y}$ in the Urinary Bladder," Cancer Biother. Radiopharm., 18[3], 413-419 (2003).

7) C. Hindorf, M. Ljungberg, S-E. Strand, "Evaluation of parameters influencing S values in mouse dosimetry," J. Nucl. Med., 45, 1960-1965 (2004).

8) M. G. Stabin, T. E. Peterson, G. E. Holburn, M. A. Emmons, "Voxel-based mouse and rat models for internal dose calculations," J. Nucl. Med., 47, 655-659 (2006).

9) A. Bitar, A. Lisbona, P. Thedrez, C. S. Maurel, D. L. Forestier, J. Barbet, M. Bardies, "A voxel-based mouse for internal dose calculations using Monte Carlo simulations (MCNP)," Phys. Med. Biol., 52, 1013-1025 (2007).
10) R. Taschereau, A. F. Chatziioannou, "Monte Calro simulations of absorbed dose in a mouse phantom from 18-flurorine compounds," Med. Phys., 34, 1026-1036 (2007).

11) E. Larsson, S-E. Strand, M. Ljungberg, B-A. Jonsson, "Mouse S-factors based on Monte Carlo simulations in the anatomical realistic Moby phantom for internal dosimetry," Cancer Biother. Radiopharm., 22, 438-442 (2007).

12) A. Mohammadi, S. Kinase, "Monte Carlo simulations of photon specific absorbed fractions in a mouse voxel phantom," Prog. Nucl. Sci. Tech., 1, 126-129 (2011).

13) T. Xie, G. Zhang, Y. Li, Q. Liu, "Comparison of absorbed fractions of electrons and photons using three kinds of computational phantoms of rat," Appl. Phys. Lett., 97[3], 033702 1-3 (2010).

14) G. Zhang, T. Xie, B. Hilde, Q. Liu, "Development of a rat computational phantom using boundary representation method for Monte Carlo simulation in radiological imaging," Proc. IEEE, 97, 2006-2014 (2009).

15) P. H. R. Peixoto, J. W. Vieira, H. Yoriyaz, F. R. A. Lima, "Photon and electron absorbed fractions calculated from a new tomographic rat model," Phys. Med. Biol., 53, 5343-5355 (2008).

16) T. Xie, D. Han, Y. Liu, W. Sun, Q. Liu, "Skeletal dosimetry in a voxel-based rat phantom for internal exposures to photons and electrons," Med. Phys., 37, 2167-2178 (2010).

17) M. A. Keenan, M. G. Stabin, W. P. Segars, M. J. Fernald, "RADAR realistic animal model series for dose assessment," $J$. Nucl. Med., 51[3], 471-476 (2010).

18) S. Kinase, "Voxel-based frog phantom for internal dose evaluation," J. Nucl. Scie. Tech., 45[10], 1049-1052 (2008).

19) S. Kinase, "Monte Carlo simulations of photon absorbed fractions in a frog phantom," Proc. IEEE, 97, 2086-2097 (2009).

$20)$ W. R. Nelson, H. Hirayam, D. W.O. Rogers, The EGS4 code system, SLAC-265 (1985).

21) S. Kinase, M. Zankl, J. Kuwabara, K. Sato, H. Noguchi, J. Funabiki, K. Saito, "Evaluation of specific absorbed fractions in voxel phantoms using Monte carlo simulation," Radiat. Prot. Dosim., 105[1-4], 557-563 (2003).

22) M. G. Stabin, M. Konijnenberg, "Re-evaluation of absorbed fractions for photons and electrons in spheres of various sizes," J. Nucl. Med., 41[1], 149-160 (2000).

23) M. Cristy, K. F. Eckerman, Specific absorbed fractions of energy at various ages from internal photon sources, ORNL/TM-8381: Vol. 1-7, Oak Ridge National Laboratory (ORNL) (1987).

24) B. Dogdas, D. Stout, A. F. Chatziioannou, R. M. Leahy, "Digimouse: a 3D whole body mouse atlas from CT and cryosection data," Phys. Med. Biol., 52, 577-587 (2007).

25) National Institute of Standards and Technology, Photon interaction cross section library, DLC-136/PHOTX (1989).

26) International Commission on Radiation Units and Measurements, Stopping Powers for Electrons and Positrons, ICRU Report 37 (1984).

27) A. Endo, Y. Yamaguchi, Compilation of nuclear decay data used for dose calculation revised data for radionuclides listed in ICRP publication 38, JAERI-Data/Code (2001-2004). 